\title{
«PARA QUE YO PUDIERA AMARTE / VIRGINIA WOOLF TUVO QUE ESCRIBIR ORLANDO": LA ESCRITURA AUTOBIOGRÁFICA DE CRISTINA PERI ROSSI
}

\author{
Ana Isabel Zamorano Rueda \\ UNED \\ aizamorano@flog.uned.es
}

\section{RESUMEN}

Este ensayo se centra en el estudio de la escritura autobiográfica de la escritora uruguayo-española Cristina Peri Rossi, quien, en 2020, publica La insumisa, volumen propiamente autobiográfico aunque su obra, tanto poética como narrativa, es una exploración de un devenir vital donde lo autobiográfico, traspasando la frontera que separa a los géneros literarios, tiene una presencia importante. Este trabajo se centra en la articulación de la vivencia personal del deseo y el amor lesbiano y, para ello, establece una conexión, dentro del «intertexto lesbiano», con otras autoras que con anterioridad y simultáneamente han intentado inscribir un deseo en el lenguaje simbólico que, hasta finales del siglo XIX, no conocía su nombre. Partiendo de los versos de Peri Rossi, nos adentramos en el entramado científico que construye narrativas como el Orlando (1928), de Virginia Woolf, y conectamos este comienzo de una articulación del amor lesbiano a la escritura autobiográfica de Peri Rossi.

Palabras Clave: autobiografía, intertexto lesbiano, discurso científico, teoría feminista, Virginia Woolf, Cristina Peri Rossi.

"PARA QUE YO PUDIERA AMARTE / VIRGINIA WOOLF TUVO QUE ESCRIBIR ORLANDO": CRISTINA PERI ROSSI'S AUTOBIOGRAPHICAL WRITING

\section{Abstract}

This article is built around the autobiographical attempts of Uruguayan-Spanish writer Cristina Peri Rossi, who, in 2020, publishes La insumisa, a volume that can be properly called autobiography although her ouvré at large and her poetical works in particular can be seen as unique autobiographical fictions as Peri Rossi's literary production at large transgresses the boundaries that divide the literary genders to express her vital experience. This work pays attention to the articulation of lesbian desire and love and, in order to do so, departs from the lines that appear in its title establishing a connection, within the "lesbian context," with other women writers who, in the past and simultaneously, have tried to inscribe in the symbolic language a lesbian desire unnamed until the end of the $19^{\text {th }}$ century. Thus, this essay provides a little incursion into the scientific discourse that builds up narratives such as Virginia Woolf's Orlando (1928) linking this discourse with Cristina Peri Rossi's autobiographical writing.

KEYwORDs: autobiography, lesbian intertext, scientific discourse, feminist theory, Virginia Woolf, Cristina Peri Rossi. 
Quizás tengas el cabello mojado el teléfono a mano que no usas para llamarme para decirme esta noche te amo (Cristina Peri Rossi, «No quisiera que lloviera», 1976)

El débito personal que Cristina Peri Rossi siente hacia Virginia Woolf lo expresa, entre otros escritos, en unos versos del poema "Historia de un amor", en el poemario Aquella Noche (1996), donde se hace explícita su deuda literaria con la escritora británica, a quien considera parte de su tradición: «Para que yo pudiera amarte / Virginia Woolf tuvo que escribir Orlando» (Peri Rossi 148). Al utilizar estos versos como la primera parte del título de este artículo dedicado a la escritura autobiográfica de Cristina Peri Rossi, hemos de subrayar, en primer lugar, que esta tradición no se refiere al personaje histórico, sino a su escritura. Es el acto de escritura, que Virginia Woolf escribiera Orlando, A Biography (1928), lo que hace posible, en espacios y tiempos tan distantes y distintos, que escriba otra mujer, Cristina Peri Rossi. Que sea la escritura y no el personaje histórico es importante pues permite, por medio de la infinita miríada de lecturas posibles, la activa interpelación a Virginia Woolf, quien, como vemos en los versos que abren este ensayo, es fundamental para que la poeta pueda amar.

El impacto más inmediato de estos versos es que inscriben parte de la producción literaria de Peri Rossi (sin duda también la de Virginia Woolf) en lo que Eleaine Marks denominó «el intertexto lesbiano» (Marks 271), referido a un espacio donde mujeres que aman a otras mujeres han inscrito en el lenguaje su amor y deseo. Definitivamente, la cuestión del Eros (y del Thanatos, pues amor y muerte están fuertemente imbricados en la escritora uruguaya) se constituye en una de las preocupaciones recurrentes que encontramos en la producción literaria de Cristina Peri Rossi. No se trata de un amor romántico el que explora Peri Rossi en su escritura, sino un amor que cobra vida a través de los sentidos y el goce de los cuerpos. Esto es así hasta el punto de que, como ha señalado Lil Castagnet en su Prólogo a la antología La barca del tiempo (2016), la escritora mexicana Elena Poniatowska menciona en diversas ocasiones que «cada vez que leo a Cristina Peri Rossi me dan ganas de hacer el amor. Leerla es una invitación al placer» (7).

Peri Rossi entiende que la clave de la indagación en el amor y el deseo se encuentra en poder escapar de la mismidad con que se articula el hecho amatorio. Nos referimos aquí a la expresión "te amo» o «te quiero» con que las amantes expresan la profundidad del sentimiento. La mismidad que encontramos en Peri Rossi ha preocupado a otras escritoras lesbianas. Por ejemplo, la británica Jeannette Winterson, en Written on the Body (1993), pone en boca del personaje ambiguo y sin nombre que protagoniza la historia las siguientes palabras: "I love you' is always a quotation" para continuar lamentando la falta de originalidad al articular la emoción del hecho amoroso: «It's the clichés that cause the trouble» (Winterson 10). Esta mismidad con que un sentimiento tan profundamente individual que ha de confor- 
marse con el desarraigo de ser expresado por el cliché «te amo» interpela de forma imperativa a la escritura que se resiste a someterse a esta condición subalterna. De este modo, la escritora uruguaya confronta el hecho de que, más que poder explorar el deseo y el amor, se ha de explorar el lenguaje del deseo y el problema de la representación en la escritura del amor.

Cristina Peri Rossi, en los versos que acompañan al título de este ensayo, nos sitúa en la paradójica relación existente entre el amor y su articulación verbal, en particular en la encrucijada donde encontramos el interrogante sobre la naturaleza del amor y una articulación plausible del deseo lesbiano en el lenguaje. Esta cuestión es uno de los hilos que hilvana la textura del lenguaje poético, en el sentido kristevano del término, que la poeta explora. La consciencia de una necesidad de búsqueda de la palabra en su rica ambigüedad pero sin el desgaste del uso, máxime cuando no existen referentes directos en el sistema heteronormativo y patriarcal de la cultura occidental, se hace fundamental en la producción poética de la escritora. Como ella ha comentado refiriéndose a la escritura de Estrategias del deseo (2004):

está escrito ya desde una visión en que se sabe que el deseo es circular, que vuelve y se va, y que cada vez que vuelve tiene algo de esa imagen en el espejo que alguna vez estuvo, de esa pregunta que uno siempre se hace: '‘a quien le estoy diciendo te quiero?' (Pérez Fondevila 181).

De forma ineludible, la estrategia a la que alude este poemario pasa por la búsqueda de significados para la expresión «te amo» que sean distintos a los asimilados al cliché que ha sido utilizado por una tradición amatoria al uso que, hasta finales del siglo XIX, no tuvo en cuenta a la mujer como sujeto hacedor, como amante y poseedora de su propio deseo. Por ello, y como la propia autora comenta en la entrevista con Pérez Fondevila, se ha de mirar a este cliché, aceptado por todas y todos como expresión última del sentimiento amoroso, desde diversos ángulos: «en el terreno de lo paradójico y de lo ambivalente y de lo que puede ser contradictorio ("te amo, no te amo", "te quiero, no te quiero", "te deseo, no te deseo"), quiero que esa reflexión sea permanente y abordable por muchos lugares» (Pérez Fondevila 188-189).

Los versos de Peri Rossi que hemos tomado para la primera parte del título se cargan, en este sentido, de significado pues nos transportan a un locus temporal, la publicación de Orlando, A Biography en 1928, que constituye un punto de inflexión en la articulación del deseo y el amor entre mujeres. Es muy importante, para entender la búsqueda que referimos en la propia experiencia amatoria de Peri Rossi, subrayar el hecho de esta traslación literario-temporal. El Orlando de Woolf constituye una articulación de una experiencia amatoria diferente, la del amor entre mujeres; esta experiencia consigue escapar de la censura. En este año, 1928, se publicaron tres novelas más cuya narrativa está construida en torno a la homosexualidad femenina, El hotel, de Elizabeth Bowen; Mujeres Extraordinarias, de Compton Mackenzie, y El almanaque de las mujeres, de la escritora norteamericana Djuna Barnes y publicado en Francia. Todos estos libros son el resultado de los cambios en el discurso científico con respecto a la sexualidad de las mujeres y del advenimiento y 
consolidación de lo que se dio en llamar «la nueva mujer» (en contraposición con la visión de ángel del hogar que se tiene de la mujer victoriana).

Por tanto, la reverberación temporal de los versos de Peri Rossi nos trasladan al momento, finales del siglo xix y principios del xx en Gran Bretaña (y también en Europa y los Estados Unidos), en el que aquello que hasta entonces no tiene nombre propio, el deseo lesbiano, comienza a articularse. Así, Peri Rossi encuentra en este momento el punto de inflexión donde comenzar la tarea de explorar su propia sexualidad, su deseo y su amor desde perspectivas diferentes. No se trata, como ha argumentado Terry Castle en su antología The Literature of Lesbianism: A Historical Anthology from Ariosto to Stonewall (2003), de que el amor entre mujeres haya sido un tema desconocido. Ciertamente, como se muestra en esta antología, se ha representado profusamente en la literatura. Sin embargo, el punto de cambio reside en que, por vez primera, la mujer se ve reflejada en discursos que hasta ahora la ignoraban, como el científico o el judicial, en relación con su propia sexualidad como sujeto y no como objeto del deseo masculino, que es el que ha prevalecido en la representación de las relaciones entre mujeres dentro del patriarcado.

Lo que parece claro atendiendo a los distintos capítulos que aparecen tanto en la antología mencionada como en otras - por ejemplo, la pionera Chloe Plus Olivia: An Anthology of Lesbian Literature from the 17th Century to the Present (1994), de Lillian Faderman- es que, desde las postrimerías del siglo XIX, y cuanto más nos acercamos al tiempo presente, las mujeres escritoras se hacen más visibles y activas en la tarea de dar un significado distinto al cliché que arriba mencionábamos. La importancia estriba no tanto en el hecho sino en la palabra, en la búsqueda de un lenguaje que represente el deseo homoerótico femenino sin necesidad de recurrir al relato masculino para encontrar una representación satisfactoria de este deseo amoroso.

Cristina Peri Rossi hace suya esta búsqueda de la palabra y de una genealogía propia, y, así, en el poema «Historia de un amor», continúa:

\section{Para que yo pudiera amarte \\ Virginia Woolf tuvo que escribir Orlando \\ Y Charles Darwin \\ viajar al Río de la Plata (Peri Rossi 148).}

Estos versos, que mencionan a Darwin y el Río de la Plata, no solo conectan Europa y América como una alegoría de la procedencia de las protagonistas de los versos en esta historia de amor aludida; además, conectan, en referencia a lo dicho hasta ahora, con el punto de inflexión que supone para la literatura memorística la erupción en la cultura occidental, en los ańos finales del siglo xix y comienzos del $\mathrm{xx}$, del tema de la homosexualidad femenina dentro de cuestiones más generales como fueron la llamada "Woman Question» (la cuestión de la mujer) y la exploración de la sexualidad de las mujeres (hasta entonces desconocida y negada por el discurso patriarcal), como hemos insinuado anteriormente.

Por primera vez en la historia, el conocimiento científico se ocupa -casi se empecina dentro de un vórtice de discursos surgidos a partir de la reivindicación por el voto y la igualdad de las mujeres- de desmentir la creencia, tenida por válida 
e inamovible, acerca de la inferioridad manifiesta de la mujer, así como intenta dar autoridad a la "norma» (aquello que es normal es aceptable y por tanto deseable) y sus desviaciones (aberraciones de la norma que en algunos casos se han de tolerar pero que nunca se han de convertir en un ideal). Unido a discursos legales, morales y políticos, el conocimiento científico del momento se fija en la mujer para refutar, en gran medida, los contenidos del discurso de los movimientos sufragistas y neutralizar el ascenso de la New Woman: esa nueva mujer, independiente económica y culturalmente, que aparece en este final de siglo y que es objeto de burlas y escarnio en revistas de gran circulación como Punch (1842-1992). A esta figura de nueva aparición en el entramado social se unen la del decadente y el dandy, quienes, de manera distinta pero con igual peligro, retan las certezas monolíticas e inamovibles del entramado sociocultural y económico de la decimonónica cultura patriarcal sostenidas en torno a la sexualidad y las diferencias de los sexos y los papeles genéricos.

Las teorías de Darwin, sin duda, significaron un cambio de paradigma en la aproximación científica a la realidad y su fenomenología, que antes era explicada como natural o debida al designio divino. Este cambio afecta a todas las áreas del saber y también a la forma de expresión artística, produciendo cambios que se desarrollarán en su plenitud en el periodo de entreguerras mundiales. La creciente confusión generada por la irrupción de la mujer en la esfera pública desdibuja las, hasta el momento, claras fronteras divisorias entre los papeles de género y sexuales; por ello, una gran parte de la investigación científica del momento se centra en restaurar el orden patriarcal al uso, un orden que requiere, entonces, de la separación entre hombres y mujeres y las esferas en que éstos y éstas se mueven. Así, la ciencia se adentra en la investigación de las características empíricamente observables que diferencian a hombres y mujeres. De este modo se intenta, con conclusiones científicas «irrefutables», restaurar la premisa de supremacía del hombre sobre la mujer. Los estudios en las ciencias sociales y la antropología de finales del siglo xix y principios del xx (los de Maine, McLennan, Spencer, Morgan, Lubbock, y Bachofen, entre otros) intentan, pues, justificar las estructuras sociales patriarcales mediante análisis históricos y evolutivos que demuestran la bonanza «natural» del papel desempeñado por la mujer desde tiempos inmemoriales.

Esta cuestión también llega a los tratados de biología y medicina científica con trabajos como The Evolution of Sex (1889), de Geddes y Thomson, quienes, en línea con lo expuesto por Spencer y por Darwin, afirman que la parte femenina de la especie humana es un caso de arrested development, término acuñado para referir, desde la antropología y la medicina, la condición de un individuo o una especie que ha llegado a su grado máximo de evolución posible. De este modo, Geddes afirma en un estudio posterior: "What was decided among the prehistoric Protozoa cannot be annulled by an Act of Parliament» (Geddes 286), invalidando, con argumentos supuestamente científicos y observables, los esfuerzos de emancipación de la lucha de las sufragistas.

Otro aspecto de gran importancia para el tema del intertexto lesbiano, que aquí nos ocupa en relación con la escritura autobiográfica de Cristina Peri Rossi, es que este periodo ve nacer una nueva rama del saber científico especializado en el 
comportamiento sexual de los seres humanos. Nos referimos a la sexología. Emergida en un contexto de cambios devenidos por la epidemia de sífilis y de otros grandes escándalos sexuales del momento, la sexología centra su estudio e investigación en demarcar de forma clara la línea divisoria entre un comportamiento aceptable y lo aberrante. La ansiedad generalizada y el menoscabo de los valores morales victorianos -toda vez que la aparente paz de la esfera privada del hogar se ve contaminada por el escándalo y la enfermedad procedentes de la esfera pública- se traducen en la petición de una legislación más dura que pueda salvaguardar a la familia de la amenaza de la depravación de comportamientos que están cada día más abiertos al escrutinio de la prensa y del público en general (mujeres incluidas). Éste es el caso, por ser conocido, del juicio a Oscar Wilde en 1895, y la atroz sentencia en la que éste concluye. Este asunto atrajo la atención de la opinión pública hacia la homosexualidad, un tema hasta entonces inexistente en lo social por estar silenciado. Si bien es generalmente ignorado por el público (no se habla de ello), no es menos cierto que la presencia del homosexual, sus lugares de reunión, y su clara distinción en comportamiento y vestimenta, son cada vez más visibles y quizá esto explique la severidad con que se juzga al escritor irlandés. Lo que hemos de señalar aquí es que este juicio marca un hito no solo por lla injusticia y severidad del castigo, sino porque es uno de los primeros juicios en los que el saber científico y el orden civil se unen para poder discriminar, de forma «objetiva», entre lo aceptable y lo que no lo es, lo aberrante.

La palabra «homosexual» se emplea en este momento por primera vez (Woods 1994) ${ }^{1}$. Desde una mirada actual, capaz de discernir tantas formas de identidad sexual, sorprende la mezcolanza de categorías ('homosexualidad', 'inversión sexual', 'tercer sexo' o 'uranismo', entre otras) disponibles en el momento histórico demarcado por los versos de Cristina Peri Rossi, entre los viajes de Darwin y la escritura del Orlando, de Virginia Woolf -sorprende sin duda a lectores y lectoras del presente, quienes acostumbramos de diferenciar entre estas categorías y muchas otras posibles y formadoras de distintas identidades sexuales-. Sin duda, la publicación de varios trabajos científicos sobre la conducta sexual no solo generó cierto alboroto sino que, sobre todo, hizo de ésta un tema de conversación generalizado por el que se empieza a cuestionar todo aquello que permanecía hasta ahora incuestionable. El estado de alboroto generalizado ante temas relacionados con la conducta sexual hace que se comience a hablar de estos temas; a ello contribuyen trabajos como los del médico alemán Richard von Krafft-Ebing, quien en Psychopathia Sexualis (1886) clasifica las entonces consideradas "perversiones» sexuales; junto con este volumen, aparecen otros más amables con las diferencias, como Sexual Inversion (1897), de Havelock Ellis, o el estudio de Edward Carpenter The

1 Woods explica que el término "homosexual» $\mathrm{y}$ «homosexuality» aparecen por primera vez en alemán, en dos panfletos escritos en Kertbeny en 1869, pero es Krafft-Ebing el autor que les da una mayor circulación cuando se traduce su tratado (Psychopathia Sexualis) primero al inglés por Charles Gilbert Chaddock, quien usa en esa traducción la palabra homosexuality en 1892. 
Intermediate Sex (1915), que describe el comportamiento «normal» de un hombre «masculino» y de una mujer "femenina» y los papeles que cada uno asume según su conducta. Así, a pesar de legisladores y de una gran parte de los científicos, este discurso regulador de la «normalidad» y sus desviaciones se convierte en el relato de una clase de amor nunca nombrado, mucho menos narrado, que ahora, desde la modernidad, sale por primera vez a la superficie. Con Foucault en mente (History 101), puede argumentarse que estos tratados y legislaciones obsesivas permiten a los grupos marginales, entre los que se encuentran las homosexuales, apercibirse de su diferencia y articularla. Emerge, de este modo, una subcultura que comienza a reivindicar y a reclamar sus derechos; un intertexto lesbiano, de narrativas acerca del amor entre mujeres, muchas desde la culpa, desde luego, pero otras no tanto, pero logrando todas ellas poner palabras, todas ellas aportando, para aquello que no podía describirse en el pasado.

Es en este contexto en el que la escritura de Orlando y su relato de un amor entre mujeres al comienzo del siglo xx nos proporciona un punto de inflexión en la articulación identitaria de mujeres que aman a otras mujeres recogida en el intertexto lesbiano. Conviene detenerse, como hemos hecho, en la importancia de estos versos, y en en su enorme significado a la hora de explorar el sujeto lesbiano en la escritura de Peri Rossi, pues su entramado sirve de andamiaje de un lenguaje que aleje su experiencia diferencial de la mismidad implícita en la figura (intertexto) de la lesbiana. En sus propias palabras:

Cada uno con su verdad solito, sin hacer la síntesis... Esto ha sido algo bastante característico de la cultura en los últimos siglos: cada sistema ha pretendido explicar todo el mundo, cosa que sería completamente tranquilizadora si pudiera hacerse. Pero el mundo es demasiado variado y los seres humanos tenemos tan pegado lo uno a lo otro que separarlo, aunque sea sólo para estudiarlo, es también reduccionista (Pérez Fondevila 189).

Como ha comentado la escritora uruguaya en varias entrevistas, no podemos tener ni tan siquiera una aproximación a la totalidad del yo sin atender a sus partes, y es por ello que Peri Rossi otorga gran importancia a otros saberes, que también nos conforman y nos condicionan como sujetos en el lenguaje, tales como la biología, la teoría evolutiva, la medicina, la antropología, el psicoanálisis, entre otros. Como la propia autora nos dice, estos saberes nos interpelan como individuos.

Si bien la obra de Cristina Peri Rossi está fuertemente impregnada de un componente autobiográfico, la escritura de la experiencia vital como acto consciente se ha dilatado en el tiempo; no es hasta el año 2020, cuando se publica La insumisa, que la autora de origen uruguayo publica un libro autobiográfico propiamente dicho. Esto puede deberse a que, como ella misma ha comentado en múltiples ocasiones, Peri Rossi no cree que haya una distinción clara entre los géneros literarios, por lo que constantemente traspasa la línea divisoria entre poesía y narrativa y entre ficción y autobiografía. La cuestión de la sexualidad es, sin duda, una de las más destacables preocupaciones de la autora. Sin embargo, su obra está marcada por otros temas personales, que señalan su devenir vital y que, indudablemente, cohabitan en la persona de Cristina Peri Rossi. Entre ellos 
encontramos grandes temas como el exilio, la política o la relación de la infancia con el mundo adulto.

Ya en el año 2005, la escritora afincada en Barcelona desde 1972 hace un comentario acerca de una autobiografía que está preparando. Se trata, según dice, de una ficción autobiográfica, consciente de lo difícil que resulta entresacar una verdad de los bosquejos de memoria de lo vivido. Así, en en el poema "Estrategias de Deseo», publicado en el poemario del mismo título (2004), nos advierte de que "La verdad nos es decible(sic)» (Peri Rossi 184, énfasis en el original). La verdad nos es impuesta y es imposible llegar a decir la verdad más allá de la que reverbera en la palabra, en el lenguaje poético, que es el más ambiguo y el más alejado del pragmatismo del lenguaje científico del que, en este caso, parte. Tal y como hemos venido argumentado, la estrategia de articulación del deseo elegida por la autora toma forma, en parte, gracias al discurso de los psicólogos y los sexólogos que a finales del siglo XIX y comienzos del xx centraron su atención en la sexualidad de la mujer lesbiana.

Cristina Peri Rossi nace en Montevideo, en 1941. Poeta, narradora, ensayista y traductora, su primera publicación es una colección de relatos, Vivencias (1963), a la que siguió Los museos abandonados (1968). Como comenta el escritor argentino Salvador Biedma en su reseña en red de la antología poética La barca del tiempo (2016), la escritora es más conocida en Uruguay por su narrativa. Sin embargo, se la considera, tanto por su poesía como por su narrativa, como una de las autoras más importantes de la lengua española. La obra de Peri Rossi ha sido traducida a varias lenguas, incluyendo el yiddish y el coreano. Esta fama, que consolida a Peri Rossi en el plano internacional, ha sobrepasado el mundo editorial para incorporarse al del merchandise, donde autoras y autores ven su nombre impreso no solo en las portadas de los libros, sino en todo tipo de objetos que se convierten en artículos que venden bien y dan buenas ganancias a los proveedores. Esta incorporación es descrita de forma cómica y magistral por la propia Peri Rossi en uno de sus poemas, el titulado «I love Cristina Peri Rossi», publicado en Playstation (2009), en el que a partir de ese toque tragicómico, típico de su producción literaria, con el que aborda la herida del amor, la importancia del deseo y el estigma de la soledad, nos describe la compra de una camiseta con un corazón dibujado y su nombre -de ahí el título del poema- en una popular tienda online. Después de dilucidar acerca de la importancia de este acto de popularización de Cristina Peri Rossi como icono, la escritora concluye el poema con un sorpresivo y, sin duda, irónicamente divertido verso en el que, al reparar en la talla que ha comprado, la voz poética nos manifiesta, desconsolada, como es costumbre en la totalidad de su obra de ficción autobiográfica: «A mí, mi amor me queda grande» (Peri Rossi 253).

Peri Rossi ha escrito de forma profusa y profunda sobre la revelación de desgarro que es el exilio; en La insumisa, por ejemplo, describe cómo recala en el puerto de Barcelona en 1972 por azar y por hastío del viaje: «¿Qué sabía yo de Barcelona cuando me subí al Cristóforo Colombo y en lugar de desembarcar en Génova, su destino, me quedé en Barcelona?» (Peri Rossi 91). Esta llegada fortuita se convierte en una residencia casi permanente hasta que en 1975 adquiere la nacionalidad española, nacionalidad que hoy en día comparte con la uruguaya. No ha retornado a su país natal porque, como ha comentado en varias entrevistas, prefiere no cambiar la 
nostalgia de Montevideo (ya conocida) por la de Barcelona. Ha recibido numerosos premios y distinciones; destacaremos tan solo algunos, los de mayor relevancia. Cristina Peri Rossi fue la primera mujer en ser galardonada con el prestigioso Premio Loewe de Poesía, en 2008, por Playstation, poemario que la autora ha calificado como completamente autobiográfico. Anteriormente recibió el Premio Ciudad de Barcelona por Babel Bárbara, en 1990. Ha sido galardonada con el Premio Internacional de Poesía Rafael Alberti, en el año 2000, y, en el 2013 recibe el Premio Don Quijote de Poesía por Estrategias de deseo. En el año 2019 se reconoce toda su obra al serle otorgado el Premio Iberoamericano de Poesía José Donoso. En 2013, el Gobierno uruguayo distingue a la escritora con la Medalla Delmiri Agustini a la Actividad Cultural.

Cristina Peri Rossi es una escritora prolija. La nave de los locos (1984), quizá su mejor novela, propone la cohabitación imposible de dos planos, el orden y el caos, que coexisten en la exploración del sentimiento de alienación del exiliado y la barbarie de los regímenes dictatoriales del cono sur de América. Peri Rossi ejerció, desde muy temprana edad, el periodismo y colaboró hasta el momento del exilio en su Uruguay natal con la revista Marcha. Más tarde, a su llegada a Barcelona, colaboró en Triunfo hasta la desaparición de la revista en la transición española. A partir de entonces Peri Rossi ha contribuido con sus columnas en los periódicos españoles de mayor tirada. Muchos de estos artículos han sido recopilados y reunidos en El pulso del mundo (2003), en el que la autora cuenta su periplo periodístico del siguiente modo:

Lamentablemente la revista no consiguió sobrevivir a la transición. Desde entonces he ejercido el periodismo de firma en los diarios más prestigiosos de España: El País, Diario 16, El Periódico, El Noticiero Universal, La Vanguardia, El Mundo. Desde hace trece años, además, escribo un artículo mensual para Grandes Firmas, de la Agencia Efe, que lo vende a los periódicos provinciales y en América Latina (Peri Rossi 9).

También es autora de varios ensayos entre los que se ha de destacar, también en el ámbito de la escritura de tinte autobiográfico, Julio Cortázar (2000), que más tarde desarrolla como escrito autobiográfico Julio Cortázar y Cris (2014). Este volumen, en sí mismo un crisol de géneros géneros literarios, desde el poema hasta la carta, pasando por el relato, el diálogo y la literatura de viajes, se centra en la relación personal y literaria que mantuvo con el escritor argentino, con el que convivió entre Barcelona y París.

Todo lo expuesto hasta ahora da idea de la cantidad ingente de material autobiográfico que ha producido la escritora uruguayo-española. Sin embargo, decíamos, la producción de una obra puramente autobiográfica, dentro de los cánones tradicionalmente adscritos al género tales como la narrativa de las memorias de una vida, se ha ido posponiendo a lo largo de los años. Al igual que otras escritoras, Virginia Woolf entre ellas, Peri Rossi reconoce en el género autobiográfico muchos impedimentos para la expresión de un yo femenino, cuya experiencia vital es necesariamente distinta a la de los grandes hombres; y es que la experiencia histórica de las mujeres, ausente de los anales canónicos al ocurrir en el ámbito de 
lo privado, hace necesaria una autorrepresentación diferente. Se ha de encontrar una narrativa que haga interesante la inacción y lo privado frente a la épica vida pública de los grandes hombres. En este sentido, da la impresión de que Peri Rossi haya ensayado con los géneros literarios para encontrar el vehículo que le permita encajar en el lenguaje su propia percepción de la realidad, y, al mismo tiempo, conseguir articular esta visión diferencial de lo acontecido sin comprometer el interés o la calidad literaria y poética. El problema posiciona a la escritora ante el abismo de la representación de lo privado, y no lo público, y, por ello, en los márgenes de la escritura autobiográfica.

Esta diferencia, inscribir de forma consciente su propio yo en la escritura, ha interpuesto una mirada caleidoscópica en su producción literaria, donde lo recordado se esparce por multitud de escritos. De la misma forma en que los momentos del pasado se esparcen en nuestros recuerdos, sin un orden predeterminado ni una significación predominante, como surgidos del instante, de la nada, la memoria se esparce en los poemas, los relatos e, incluso, en los ensayos que escribe Cristina Peri Rossi. Sin embargo, la autora ha sido incapaz, hasta la fecha de la publicación de La insumisa, de plasmar de manera consciente esas irrupciones de la memoria en la escritura. Henchida de ficción y de experiencia de vida, este intento consciente de escritura autobiográfica nos sorprende con un lenguaje infantil que busca aunar la inocencia y el tiempo de niñez con la experiencia de la amante adulta, quien nos narra con humor lleno de piedad y ternura su despertar sexual y su maduración en el deseo y el amor. En una reflexión sobre cómo acometer la escritura de sus memorias la poeta nos dice:

Por otro lado, para mí una novela autobiográfica tiene la ventaja, frente a las memorias, de que uno está obligado a seleccionar. No se escribe todo lo que pasó sino solo lo que a uno le parece ilustrativo acerca de la construcción de su personalidad o de su vida, aunque también te plantea problemas a la hora de decidir qué hacer con ciertas cosas: creo que voy a tratar de escribir mis memorias sin hacer como Dante, que se vengó de todo el mundo (Pérez Fondevila 191).

Los fundamentos que se han establecido tradicionalmente como intrínsecos de la escritura autobiográfica ${ }^{2}$ quedaron marcados en el trabajo de Georges Gusdorf "Condiciones y límites de la autobiografía» (Conditions and Limits of Autobiography) (1956), considerado, generalmente, como la definición más completa del género: «La aparición de la autobiografía implica una nueva revolución espiritual: el artista y el modelo coinciden, el historiador se enfrenta a sí mismo como objeto (Gusdorf 31, énfasis añadido). La autobiografía, Gusdorf continúa, «es el espejo en el que el individuo refleja su propia imagen» (33). El argumento esgrimido por Gusdorf asume el completo e idéntico reflejo del individuo en la escritura; por ello, para la recuperación del sujeto en el tiempo y el espacio bastaría con producir una escritura autorrefrac-

${ }^{2}$ La autora de este ensayo ha desarrollado estas teorías de forma más prolija en el volumen coeditado Cartografias del yo. Mujer y autobiografía (2007). 
taria. El postulado de Gusdorf, sin embargo, plantea ciertos problemas que han sido detectados por la crítica feminista y postestructuralista. La noción de la total coincidencia entre sujeto e imagen es producto de una percepción positivista del ser y de la creencia de que, en última instancia, el sujeto puede ser totalmente conocido. Como han apuntado críticas feministas tales como Shari Benstock, este planteamiento de articulación del yo en la escritura resulta, desde una perspectiva psicoanalítica, imposible ya que la coincidencia entre autor y sujeto de la autobiografía es ilusoria y basada en la presuposición de identidad entre el sujeto y su imagen en el espejo.

De acuerdo con la teoría lacaniana, es en el «estadio del espejo» cuando el niño -hablo en masculino porque así lo hace Lacan-identifica su propio yo como diferente del yo materno. En el desarrollo psicológico, el estadio del espejo precede a la sumisión del sujeto a la Ley del Padre, lo que supone la represión del Complejo de Edipo y la adquisición del lenguaje y la cultura. Lo que hay que reseñar aquí, en relación con el tema que nos ocupa, es que en el estadio del Espejo el individuo adquiere una imagen de unidad del cuerpo que es de otra manera percibido como fragmentado por los sentidos. Para Lacan, como Elizabeth Grosz explica: «el desarrollo del ego del infante depende de su habilidad para identificarse con una imagen de su unidad corporal» (Grosz 83). Sin embargo, también argumenta Lacan, la unidad del sujeto es una falsa imagen de uno mismo impuesta desde fuera en el reflejo del espejo, que además refleja el mundo que está alrededor también.

La identificación es, pues, tan solo imaginaria, pues solo podemos ver parte(s) de nuestro cuerpo, así que la otra parte hemos de incorporarla como tal; coincidir con la totalidad corporal es un imposible. No obstante, tiene que ser vivida por el sujeto como real para poder establecer una posición coherente y estable como sujeto hablante. La unidad percibida es, por tanto, asimétrica, ficticia y artificial. La unidad asimétrica del individuo está formada por dos "partes», el consciente y el inconsciente (según la terminología freudiana), o lo que Lacan denomina el «je» y el «moi», el sujeto y el objeto. El sujeto, el «je», es percibido como parcial, fragmentado y desintegrado mientras que el objeto, la imagen, se experimenta como un todo. El desarrollo psicológico del individuo tiende a la fusión, a una homogeneidad mucho más segura y confortable que reprime, por este motivo, la heterogeneidad y multiplicidad del inconsciente, lo cual sería difícil de manejar de otra manera. De modo que, como expone Shari Benstock, el inconsciente

No es la profundidad más remota del consciente [...] sino más bien una juntura interna, un espacio entre lo «interno» y lo «externo» -es el espacio de la diferencia, la brecha que el impulso hacia la unidad del sujeto nunca puede ser cerrada en su totalidad (Benstock 16).

Desde el psicoanálisis, la definición de autobiografía de Gusdorf no tiene en cuenta, por tanto, un aspecto muy importante: la asimétrica relación que existe entre el individuo y su imagen. Lo que corrobora en su teoría, ciertamente pionera al intentar definir la autobiografía como género, es que Gusdorf toma como fuente para su estudio predominantemente autobiografías escritas por hombres. En estas autobiografías existe un sistema de defensa lingüístico que, en apariencia, controla exitosamente la discrepante multiplicidad de voces del inconsciente. La ilusoria coin- 
cidencia de la entidad teleológica de la autobiografía masculina, como por ejemplo en la de san Agustín o en la de Rousseau, se basa en el firme convencimiento de que el escritor tiene autoridad absoluta sobre su subjetividad. Esto es porque, como señaló Nancy Walker, la intención última de muchas autobiografías masculinas es la de mostrar el «Gran Hombre» a sus descendientes (Walker 277-278) y, por ello, existe una tendencia en estas autobiografías a disimular los vacíos de memoria y a evitar las inseguridades y las dislocaciones de tiempo y espacio inherentes a la escritura autobiográfica. Esto es así porque, como el propio Gusdorf reconoce, existe una diferencia entre la autobiografía que describe al sujeto público de la que describe al sujeto privado: «la cuestión cambia radicalmente cuando la parte privada de la existencia asume más importancia» (Gusdorf 37). Gusdorf no ahonda en esta diferencia, la cual, argumenta Donna C. Stanton (1985), marca la diferencia entre la escritura de mujeres y la de hombres: «las narrativas masculinas eran lineales, cronológicas, coherentes mientras que las de las mujeres eran discontinuas, digresivas, fragmentadas» (Stanton 35).

Así, atendiendo a las palabras de Peri Rossi, resulta imposible alcanzar una verdad absoluta con respecto a la representación del yo expresado en Estrategias del deseo, «La verdad no es un relato» (84), por lo que ha de conformarse, al cabo del tiempo y después de años de intentos de autorrepresentación en todos los géneros posibles, con esa parte de la verdad que sí es alcanzable: la que ha de elegirse dentro de lo que la memoria nos ofrece, aquello que puede utilizarse para la escritura de una autobiografía.

Más aún, ha de tenerse en cuenta que no existe en la literatura una tradición de «autobiografía de mujer» a la que recurrir. La queja que expresa Virginia Woolf a su amiga, la compositora Ethel Smyth, en una carta fechada en 1940 -«estuve pensando, la otra noche, que no ha habido nunca una autobiografía escrita por una mujer [...] nada que pueda compararse con Rousseau» (Nicolson 453) -, es la misma de Cristina Peri Rossi, quien ha comentado en numerosas ocasiones que el mundo de los hombres es muy distinto al de las mujeres. El abismo está en no considerar lo vivido como de interés general.

El intrigante título de la autobiografía de Cristina Peri Rossi, La insumisa, nos refiere a los años rebeldes de la niñez y juventud, años anteriores al exilio (aunque hay referencias a la salida de su país natal) que resultan muy formativos para su identidad como escritora y conformadores de su deseo erótico y su sexualidad. La autobiografía abre con un primer capítulo titulado «Primer amor», donde nos narra la complicada relación que mantuvieron sus padres. El padre, casi ausente, impone su presencia de forma inesperada como forma de control: «aparecer y desaparecer sin aviso era una forma de poder» (Peri Rossi 9). Cristina Peri Rossi pronto se erige en salvaguarda de la madre contra los ataques del padre y nos sorprende cuando nos dice que, a la temprana edad de tres años, propone matrimonio a su madre. La madre, ese primer amor que titula este capítulo, no se escandaliza ni rechaza la idea, muy al contrario, incentiva a la pequeña a crecer para así poder hacer realidad esta propuesta. Este incentivo, crecer para satisfacer el deseo de estar con la madre, será una constante en Cristina Peri Rossi y marcará la búsqueda del amor y de las futuras relaciones. Tal y como nos dice la Peri Rossi adulta que escribe la autobiografía: 
Imaginaba un futuro celestial, lleno de paz y de armonía, de lecturas fabulosas, paseos apasionantes, veladas de ópera (mi madre tenía una maravillosa voz de soprano), ternura, complicidad y felicidad ¿Qué más podía pedir a una pareja? (Peri Rossi 9).

Se convierte así en la «trovadora del amor» que intentará reparar el daño causado por el padre y que nos presenta una autobiografía donde el deseo y el amor recorren transversalmente todo lo narrado. La madre descrita en la autobiografía no es una madre castradora, pues siempre impulsó el genio creativo y emprendedor de la hija, así como su independencia y originalidad. Sin embargo, impone una responsabilidad a la niña Peri Rossi, al obligarla a madurar rápido. La poeta se vio inmersa desde la infancia en una díada madre-hija de interdependencia emocional difícil de romper, incluso en la madurez: «También comprendí que podía amarla a ella y amar a otras personas, al mismo tiempo. Ella no siempre lo entendía bien» (Peri Rossi 19). Ni tan siquiera la llegada de una hermana pudo contener esta demanda emocional materno-filial. La llegada de esta hermana es despachada en la autobiografía de forma rápida y sentenciosa cuando, al conocerla después de una estancia en el campo durante el embarazo de la madre, y desencantada porque el bebé no puede ser compañera de aventuras, nos dice: «El tema hermana quedó zanjado para mí» (Peri Rossi 15). De este modo la escritora incorpora la nueva presencia de la hermana; no como un impedimento entre ella y su madre, sino como un añadido al darle a la pareja, al futuro matrimonio, el resultado «lógico» de tan estrecha relación: «Es más, cuando salíamos las tres a pasear por la ciudad, parecíamos una verdadera pareja: mi madre y yo, casadas, y la beba el fruto de nuestro matrimonio» (Peri Rossi 16).

Ante la insistencia en pedir la mano de su madre, ésta le comunica que es un matrimonio imposible, esta vez con la excusa de ser un acto prohibido. Es entonces cuando la escritura nos revela la importancia de la narración de esta primera memoria, de este primer amor. Aquí asistimos al momento en que, por primera vez, Cristina Peri Rossi se da cuenta de que hay deseos que no pueden colmarse por estar fuera de la ley: «Este conocimiento, adquirido a temprana edad, fue una de las decisiones más decisivas de mi infancia, y sus consecuencias duraron toda la vida» (Peri Rossi 18). Esta revelación queda unida, en la narrativa de vida de Peri Rossi, al deseo amoroso por otras mujeres: «agradecí mucho a mi madre que no me explicara entonces, cuando tenía tan pocos años, que no podíamos casarnos porque ambas éramos del mismo sexo» (Peri Rossi 19). La madre, a lo largo de las distintas experiencias amorosas, permanece como recuerdo de aquel primer deseo, ese amor coincidente en gustos, aficiones y pasiones que la autora recuerda como inamovible; tan solo con la edad, al revertir los papeles madre e hija, se cumple el deseo de insumisión a las leyes de los hombres.

Otra insumisión que encontramos en la autobiografía refiere cómo, desde pequeña, Peri Rossi se siente atraída por todo lo que significa aventura y movimiento. La autora nos describe su quehacer diario con anécdotas que son consideradas, en aquel momento histórico, más propias de niños que de niñas. De mal comer, un día la abuela amenaza con llevarla al asilo si no toma el café con 
leche; la niña, asustada ante la idea pero lejos de amedrentarse, se propone hacer acopio de todos los utensilios y víveres que considera pueden ayudarle a sobrevivir en esa institución:

Aunque llevaba una vida aparentemente normal -iba a la escuela, hacía los deberes, me trepaba a los árboles, intentaba la destilación de los pétalos de rosa para fabricar perfumes, me peleaba con mi hermana, odiaba a mi padre, escribía obras de teatro, escuchaba arias de ópera, intentaba fabricar pólvora con una fórmula que había encontrado en un libro, contestaba a mis mayores- nunca olvidaba que mi porvenir era el asilo y esta idea, en el fondo de mi pensamiento, me tenía muy atenta a cualquier cosa que pudiera aparecer y ser útil para mi vida futura (Peri Rossi 60).

Esta agencia ante la amenaza supone otro momento revelador de gran importancia a lo largo de la vida de la escritora: «Acababa de comprender uno de los mecanismos más crueles de los mayores: asustar a los más débiles, aterrorizarlos» (Peri Rossi 63). Esta enseñanza le será muy útil para afrontar el tiempo anterior a su partida al exilio, cuando en los 70 recibió amenazas de muerte por parte de los mecanismos represivos de la junta militar golpista. También para hacer frente a las incertidumbres y los miedos que el descubrimiento de su sexualidad en esos años de juventud le van a causar.

Con una comicidad reveladora del pánico sentido en el momento en que su amiga de colegio, Alina, con quien comparte el secreto de su amor hacia otras mujeres, le revela que son unos seres monstruosos por su deseo lesbiano, la autora introduce el diálogo en la narrativa como técnica para hacernos partícipes de la inmediatez del vórtice de ideas que le pasaron por la mente en ese momento de incertidumbre: durante el recreo, Alina llega corriendo, y gritando le revela que hay una palabra para su sentimiento, homosexualidad, y que por ser homosexuales: «iSomos anormales! ¡Somos anormales!» (Peri Rossi 216). Aturdida por la situación, por la incertidumbre creada en la paradoja de que su amor por otra mujer sea asociado a lo monstruoso, y lo injusto que siente esta situación, la escritora nos revela una nueva insumisión que ratifica su decisión de ser escritora. «Voy a ser escritora», le dice a su madre cuando intenta encontrar alguna verdad en las palabras de Alina. Así, finalmente, disiente con el orden establecido:

Si todos éramos hijos de Dios y Dios nos había creado, a mí me había creado anormal, y por tanto no era mi culpa. Yo no había pecado por serlo, de modo que no me sentía culpable. Y si moría anormal y me enviaban al infierno lo aceptaría con resignación, porque Dios me había creado así. Sentí rencor hacia Dios por primera vez en la vida, pero me parecía un sentimiento loable, de modo que decidí no volver a comulgar ni a confesarme, dado que no me sentía culpable (Peri Rossi 219).

La extrapolación del descubrimiento del sentimiento amoroso entre mujeres al plano de lo religioso es algo que se ha hecho en otras narrativas; por ejemplo, Dorothy Strachey utiliza el lenguaje religioso como una forma de expresión amorosa entre mujeres en su pequeña novela autobiográfica Olivia by Olivia (1949). De hecho, el religioso es un lenguaje al que se ha recurrido en la literatura lesbiana para poder expresar un sentimiento que, desde la culpa, sublima el deseo amoroso. 
Más aún, al sacar fuera de sí misma el sentimiento de culpa, Cristina Peri Rossi se adhiere a la corriente de confesiones que, a partir de los estudios de los psicólogos y los sexólogos finiseculares que hemos brevemente descrito en este ensayo, buscan la expiación y, aun cuando pueda ser discutido el método, proporcionan una articulación del sentimiento homoerótico entre mujeres.

Como ha argumentado Laura Freixas en su reseña de la publicación de $L a$ insumisa en el Cultural de La Vanguardia: "Un hilo conductor guía La insumisa: el amor. A la madre, a la literatura, a Uruguay, a sucesivas novias» (Freixas). Ciertamente, la diferencia temporal hace posible que lo que Vita Sackville-West describe en Retrato de un matrimonio como un estado de su personalidad «aberrante» que hubiera preferido mantener dormido, aun cuando la exaltación, el placer y la sensación de libertad que le produce son innegables y así lo muestra en su autobiografía, en el caso de Cristina Peri Rossi, la confesión desculpabilizada y la liberación que su instinto de insumisión le proporciona hacen que pueda terminar su autobiografía con el triunfo del amor entre mujeres y la consecución del deseo amoroso en ese feliz final (restringido a un momento de vida espaciotemporal en lo real) que supone el penúltimo capítulo, titulado «El beso». Es en este capítulo donde, haciendo gala de su estilo más sensual y erótico, Cristina Peri Rossi nos abre la puerta para que asistamos a su primer encuentro homerótico, ocurrido en casa de su madre cuando se encontraba con una amiga que había venido a estudiar. Este recuerdo que nos narra explícitamente el encuentro amoroso entre las dos mujeres supone, y éste es el gran logro de esta autobiografía y su originalidad, despojarse de la culpa y mostrar en el texto autobiográfico la celebración del sexo, el deseo y el amor entre mujeres. Es esta autobiografía un acto de insumisión que rompe el doliente silencio de la sexualidad femenina y la posibilidad de articular un amor que sucede fuera de la autoridad patriarcal: el deseo y el amor lesbiano. 


\section{REFERENCIAS}

Аввотт, Porter H. “Autobiography, Autography, Fiction: Toward a Taxonomy of Literary Categories”. New Literary History 19 (1988), pp. 597-615.

Benstock, Shari. The Private Self: Theory and Practice of Women's Autobiographical Writings. Chapel Hill: University of North Carolina Press, 1988.

Fariña Busto, María Jesús. «Donde habita el deseo: eros y el lenguaje poético». Erotismo, transgresión y exilio: las voces de Cristina Peri Rossi. Coord. Jesús Gómez de Tejada. Sevilla: Universidad de Sevilla, 2017, pp. 69-87.

Fariña Busto, María Jesús. «Condición de mujer. Las políticas del género en la obra de Cristina Peri Rossi», en Suárez Briones, Beatriz (et al.), Escribir en femenino. Poéticas y políticas. Barcelona: Icaria, 2000, pp. 235-248.

Fariña Busto, María Jesús. "El beso deseado de tu boca. Nombres y voces para una genealogía lesbiana [España y Portugal, primeras décadas del siglo veinte]». Investigación Feminista. Monográfico: Estudios feministas lesbianos y queer 10:1 (2019), pp. 79-96.

Foucault, Michel. The History of Sexuality. An Introduction. London, New York, Victoria, Ontario and Auckland: Penguin. 1981.

FreiXAs, Laura. «Trovadora y guardaespaldas», La Vanguardia. https://www.lavanguardia.com/ cultura/culturas/20201128/49762588608/cristina-peri-rossi-libros-feminismo.html. 28/11/2020.

Gross, Elizabeth. «The Body of Signification», en Fletcher, John y Benjamin, Andrew (eds.), Abjection, Melancholia and Love. The Work of Julia Kristeva. London and New York: Routledge, 1990, pp. 80-103.

Gusdorf, G. «Conditions and Limits of Autobiography (J. Olney, Trans.)», en Olney, James (ed.), Autobiography: Essays Theoretical and Critical. Princeton: Princeton, University Press, 1980, pp. 28-48.

Hutcheon, Linda. A Poetics of Postmodernism. History, Theory, Fiction. New York, London: Routledge, 1988.

Marks, Elaine. «Lesbian Intertexuality», en Stambolian, George y Marks, Elaine, Homosexualities and French Literature. Ithaca: Cornell UP, 1979, pp. 353-377.

MÉrida Jiménez, Rafael. «Entornos del canon de la literatura lésbica (y de las escrituras sáficas) en España». Nerter, 2 (2018), pp. 10-20.

Nicolson, Nigel. Retrato de un matrimonio; prólogo de Marta Pessarrodona; traducido por Oscar Luis Molina. Barcelona: Grijalbo (El espejo de tinta. Vidas privadas), 1989.

Nicolson, Nigel. Virginia Woolf. New York, London: Penguin Ltd., 2000.

Nicolson, Nigel y Trautman, Joanne Trautman (eds.). Leave the Letters Till We're Dead: The Letters of Virginia Woolf, VI: 1936-1941. London: Hogarth Press, 1975.

Peri Rossi, Cristina. La barca del tiempo. Antología poética. Madrid: Visor, 2016.

Peri Rossi, Cristina. El pulso del mundo, artículos periodísticos. 1978-2002. Montevideo: Ediciones Trilce, 2003.

Peri Rossi, Cristina. La insumisa. Palencia: Cálamo, 2020. 
Pérez Fondevila. «Del deseo y sus accesos: una entrevista a Cristina Peri Rossi». Lectora: revista de dones i textualitat, 11 (2005), pp. 181-194. https://www.raco.cat/index.php/Lectora/article/view/205535/284734.

Roffé, Reina. «Diferencias negociables». Conversaciones americanas. Madrid, Páginas de Espuma: 2001, 23-253.

Stanton, Donna C. «Autogynography: Is the Subject Different?», en Stanton, Donna C. (ed.), The Female Autograph. New York, 1985.

Sounami, Diana. The Trials of Radclyffe Hall. New York: Doubleday, 1999.

Weedon, Chris. Feminist Practice and Poststructuralist Theory. Oxford: Basil Blackwell, 1987.

Winterson, Jeanette. Written on the Body. New York: Knopf, 1993.

Woolf, Virginia. Orlando. A Biography. London, New York, Victoria, Ontario and Auckland: Penguin, 1993. 
